\title{
Optimization of ZigBee Network Parameters for the Improvement of Quality of Service
}

\author{
Sun Maung, Shafia Shiraje, Atiqul Islam, Mohammad Mobarak Hossain, \\ Shamsun Nahar, Md. Faizul Huq Arif* \\ Department of Computer Science and Engineering (CSE), World University of Bangladesh (WUB), Dhaka, Bangladesh \\ Email: sunmg714@gmail.com, shafiaa32@gmail.com, atiqul.chowdhury2@gmail.com,mobarak3112@gmail.com, \\ shamsun_nahar@ymail.com, *arifict27@gmail.com
}

How to cite this paper: Maung, S., Shiraje, S., Islam, A., Hossain, M.M., Nahar, S. and Arif, Md.F.H. (2018) Optimization of ZigBee Network Parameters for the Improvement of Quality of Service. Journal of Computer and Communications, 6, 1-14. https://doi.org/10.4236/jcc.2018.66001

Received: May 1, 2018

Accepted: June 2, 2018

Published: June 5, 2018

Copyright $(9) 2018$ by authors and Scientific Research Publishing Inc. This work is licensed under the Creative Commons Attribution International License (CC BY 4.0).

http://creativecommons.org/licenses/by/4.0/

\begin{abstract}
Network enabled digital technologies are offering new and exciting opportunities to increase the connectivity of devices for the purpose of home and office automation. ZigBee (IEEE 802.15.4) is such a digital wireless technology that is used for personal area networks. In this paper, an office automation network using the combination of fixed and mobile IEEE 802.15.4 has been deployed and analyzed. The QoS parameters of the network as the performance metrics like throughput, MAC delay and data dropped rate have been investigated. Finally the network has been finalized with the optimized QoS parameters.
\end{abstract}

\section{Keywords}

ZigBee, Quality of Service, WSN, Riverbed

\section{Introduction}

Nowadays wireless sensor network (WSN) technologies are growing popular for several kinds of applications. Wireless sensor network (WSN) is the self-organizing ad-hoc multi-hop network that consists of spatially distributed sensor and nodes [1]. In recent years wireless sensor networks (WSN) have been used in many areas like military, agriculture, industry, home and domestic, health and medical, environment monitoring and so on [2]. The increase of mobile computing devices in different sectors has created a demand for wireless personal area networks. To meet this challenge a WSN standard IEEE 802.15.4 was implemented by J. Zheng and M. J. Lee [3] which is named as ZigBee.

ZigBee is a wireless standard based on IEEE 802.15.4 specification for personal area network. ZigBee facilitates low data transfer rate, energy efficient, Machine to Machine (M2M) communication, secure communication and so on. The 
main point of wireless network is to send reliable data between node and network; and ZigBee makes it easy. The ZigBee network automatically figures out how to route the data from one node to another with the maximum chance of success [4]. ZigBee was designed to provide high data throughput in lower power consumption. ZigBee low power consumption limits transmission distances to 10 - 100 meters depending on power output and environmental characteristics [5]. ZigBee has three kinds of devices; ZigBee coordinator, ZigBee router, and ZigBee end device. ZigBee coordinator is the root of the network. The coordinator creates the network zone then the other nodes connected with the coordinator. ZigBee coordinator may be broken down to operate the network maintaining convenient (QoS) [6]. Several topologies like star, mesh and tree can be developed with the use of ZigBee devices [7].

Routing is an important parameter of a particular ZigBee network and it impacts on the performance of ZigBee network. In streetlight monitoring system ZigBee tree topology perform highly better than mesh topology with ACK mechanism [8]. Article [9] analyzed the performance of IEEE 802.15.4 network with 100 nodes in a Star routing method. ZigBee wireless sensor network topology gives different throughput based on different place. In underground and tunnel, mesh routing gives better performance than cluster tree routing [10]. Another study enhances the quality of service (QoS) in ZigBee network using Stochastic model and widest path model [11].

In office working, we need high speed network system. Hence performance of the office network must be good enough to adjustable with the office working speed. In addition there need fixed and mobile device for monitoring and controlling. It is essential to know how nodal mobility affects on ZigBee routing protocol. In the article [12], a simulation study has been done to evaluate the nodal mobility affects. However, there needs an extensive study on the Quality of Service (QoS) of the ZigBee network. In this regard, we have designed a ZigBee network comprising of both mobile and fixed devices. And the distance range of our ZigBee network is $100 \star 100$ meters.

In this paper, we evaluated the scenario which gives better performance in terms of throughput; data dropped rate, and MAC delay. In this research work, we tried to find out the parameters to enhance the quality of service (QoS) in office ZigBee network. In this regard we evaluate the impact of routing method, Acknowledgement (ACK) variation, packet distribution technique, and device to device variation.

\section{Research Methodology}

A literature review is performed to find out the wide range use of IEEE 802.15.4. Based on the literature review there is no published article for the combination of mobile and fixed ZigBee devices. In addition a careful study of how to enhance Quality of service (QoS) to gain better throughput with fewer data dropped rate and low MAC delay in ZigBee network has been performed. In this study, an analysis is performed to optimize the network parameters for im- 
proved Quality of Service (QoS).

A Supported simulation tool was needed to design a network model and analyze its performance. A deep study has been performed on different types of network simulation tools like Network Simulator 2 (NS2), Network Simulator 3 (NS3), OMNET++ and Riverbed. We found that Riverbed is user friendly to design and implement a network. In addition the Riverbed simulation tool provides the fastest discrete event simulation use to evaluate various network parameters on its performance [13].

Simulated data are collected from different layer of ZigBee protocol. Then the collected data are analyzed and appropriate graphs are plotted. Finally optimization of network parameters for improved QoS has been done properly and carefully.

\section{Design and Implementation}

The main objective of this simulation study is to gain high throughput, and to reduce data dropped rate as they are considered important QoS parameters of a network. Here, Riverbed Modeler version 17.5 is used for designing and implementation of ZigBee network. We have considered an office network having dimension of $100 \mathrm{~m} \times 100 \mathrm{~m}$. The designed network consists of mobile and fixed devices. The comparative analysis involves global throughput, MAC delay and data dropped rate as the parameters of QoS. In this simulation the overall scenario has been considered taking ZigBee Coordinator (Fixed), ZigBee Router (Fixed \& mobile), ZigBee end device (Fixed \& Mobile), and ZigBee end device advance (Fixed \& mobile) in every configuration. The parameters are as follows: (Table 1, Figure 1).

Table 1. ZigBee Network scenario overview.

\begin{tabular}{cc}
\hline Parameters & Description \\
\hline No. of ZigBee Coordinator (Fixed) & 1 \\
No. of ZigBeeRouter (Fixed). & 4 \\
No. of ZigBee end device (Fixed) & 8 \\
No. of ZigBee Router (Mobile) & 4 \\
No. of ZigBee end device (Mobile) & 8 \\
No. of ZigBee end device adv (Fixed) & 4 \\
No. of ZigBee end device adv (Mobile) & 4 \\
Distance range & 100 m¹00 m \\
Packet size & 1024 kbs (cont) \\
Topology & 3 \\
ACK Status & Enabled \\
Packet Interval Time & Constant (1.0) \\
Start and Stop Time & Uniform (20,21), Infinity \\
Packet Reception Power & -0.85 \\
\hline
\end{tabular}




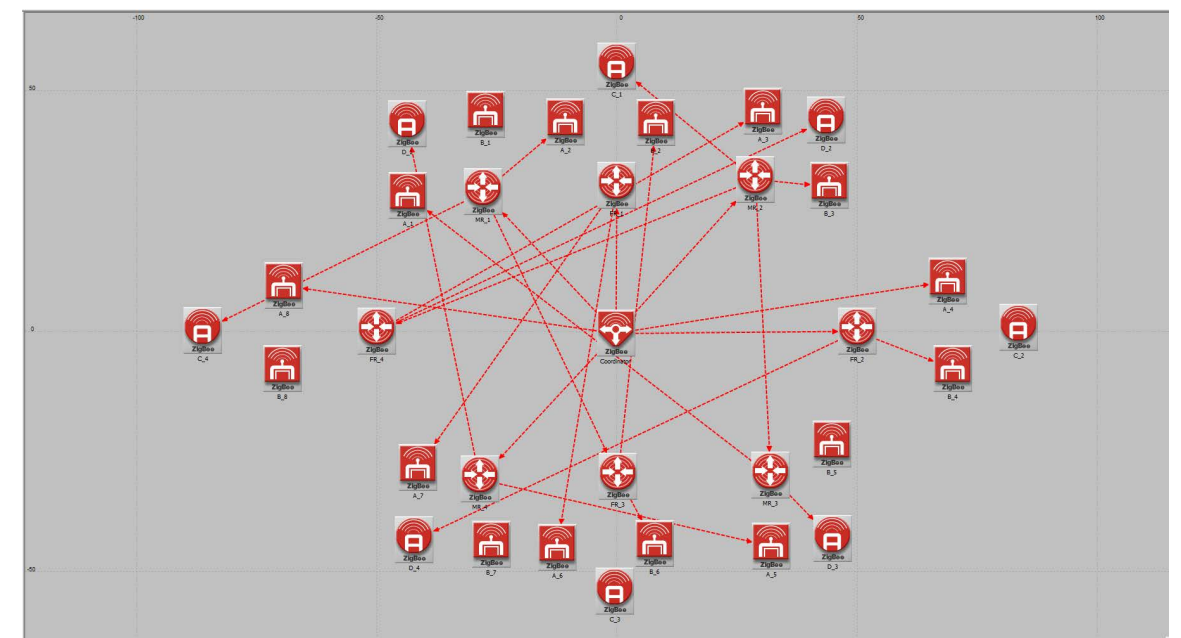

Figure 1. ZigBee network simulationscenario.

\section{Results and Analysis}

We create a ZigBee network design using some fixed and some mobile ZigBee devices for office environment. First we simply compare the three different scenarios based on three routing methods-Star, Tree and Mesh. The comparative analysis has been done for some Qos parameters like throughput, MAC delay and data dropped rate with respect to simulation. To improve the ZigBee network performance parameters of the Quality of services (QoS) we need to identify the best routing. According to the simulation result Figures 2-4 illustrate that tree routing shows better performance for our designed network.

For further analysis we selected tree routing for the designed network. In the network we took Coordinators' ACK status disable. Now we create a new scenario in which we take coordinators' ACK status enable and compare with the previous network. We can find from the Figures 5-7 that the new scenario shows better performance.

For the further investigation we selected tree routing and Coordinators' ACK status was enable in our designed network. Now we will compare between constant, poisson and uniform packet distribution technique; where packet size was 1024 Kbs. We found from the Figures 8-10 that the Poisson distribution technique shows better performance.

For the next analysis the selected parameters are: Tree routing, Coordinators' ACK status enable, and the Poisson packet distribution technique. In previous scenarios all routers' (mobile and fixed) destinations are to coordinators only. Now we will define another scenario in which the mobile routers' destinations are to all coordinators and rests of the criteria are same. Now a comparative analysis will be done between these two scenarios.

According to the simulation results (Figures 11-13) we found that the scenario in which all mobile routers' destinations are to all coordinators and routers show better performance in terms of throughput. And it shows 2.30 times better throughput than the previous scenario. However in terms of MAC delay and 


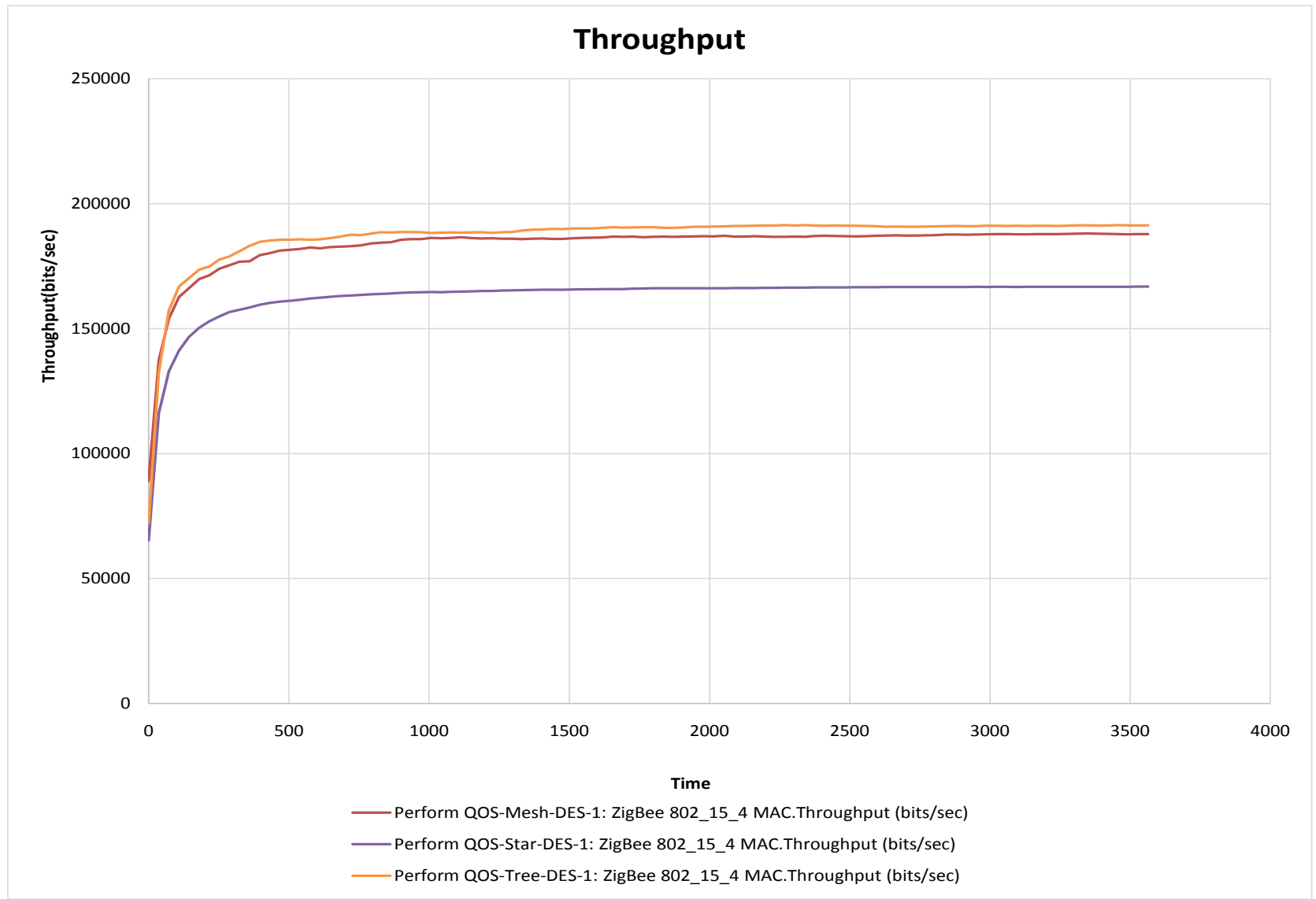

Figure 2. Throughput (bits/second) for scenario 1.

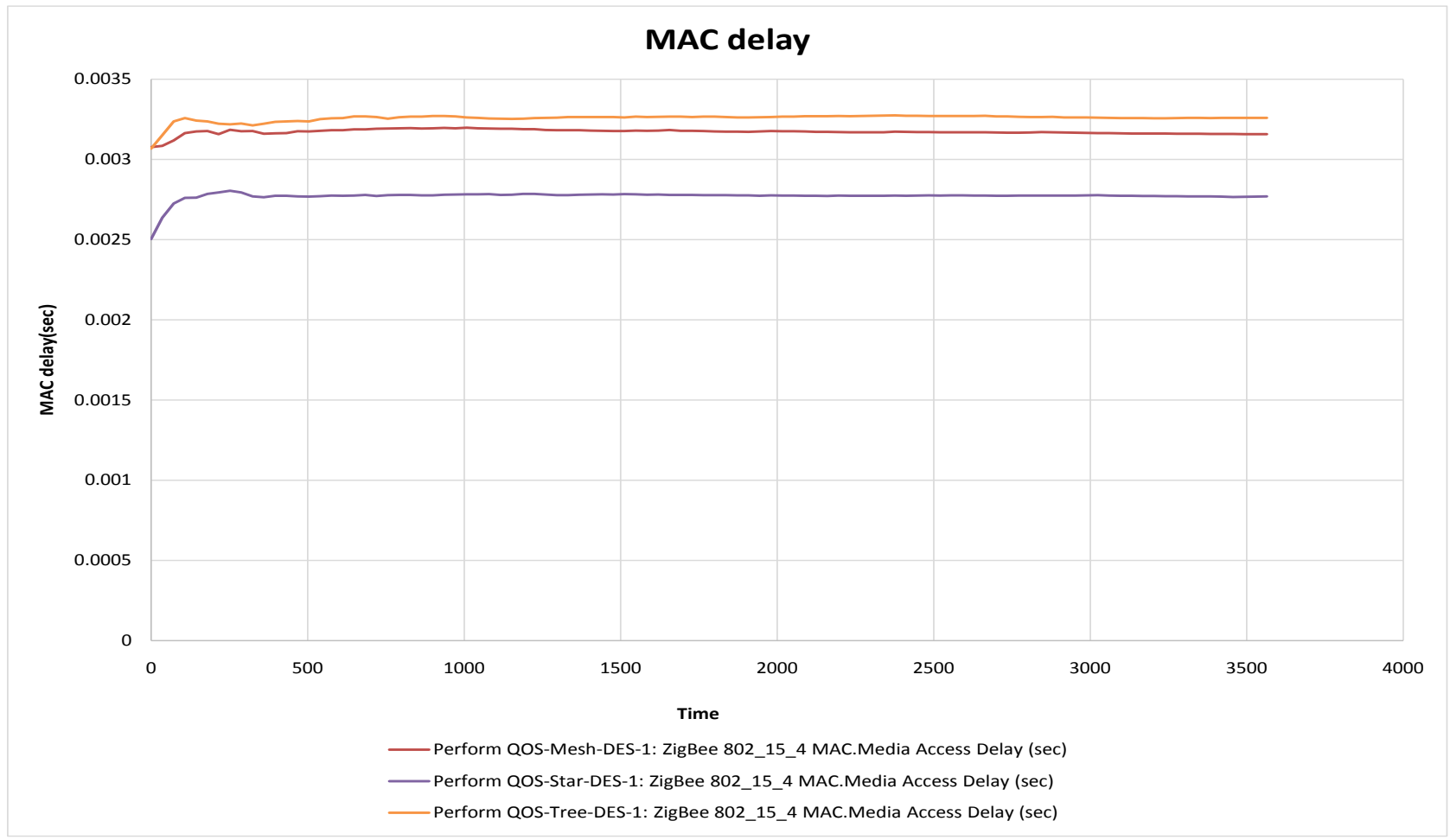

Figure 3. MAC delay (second) for scenario 1. 


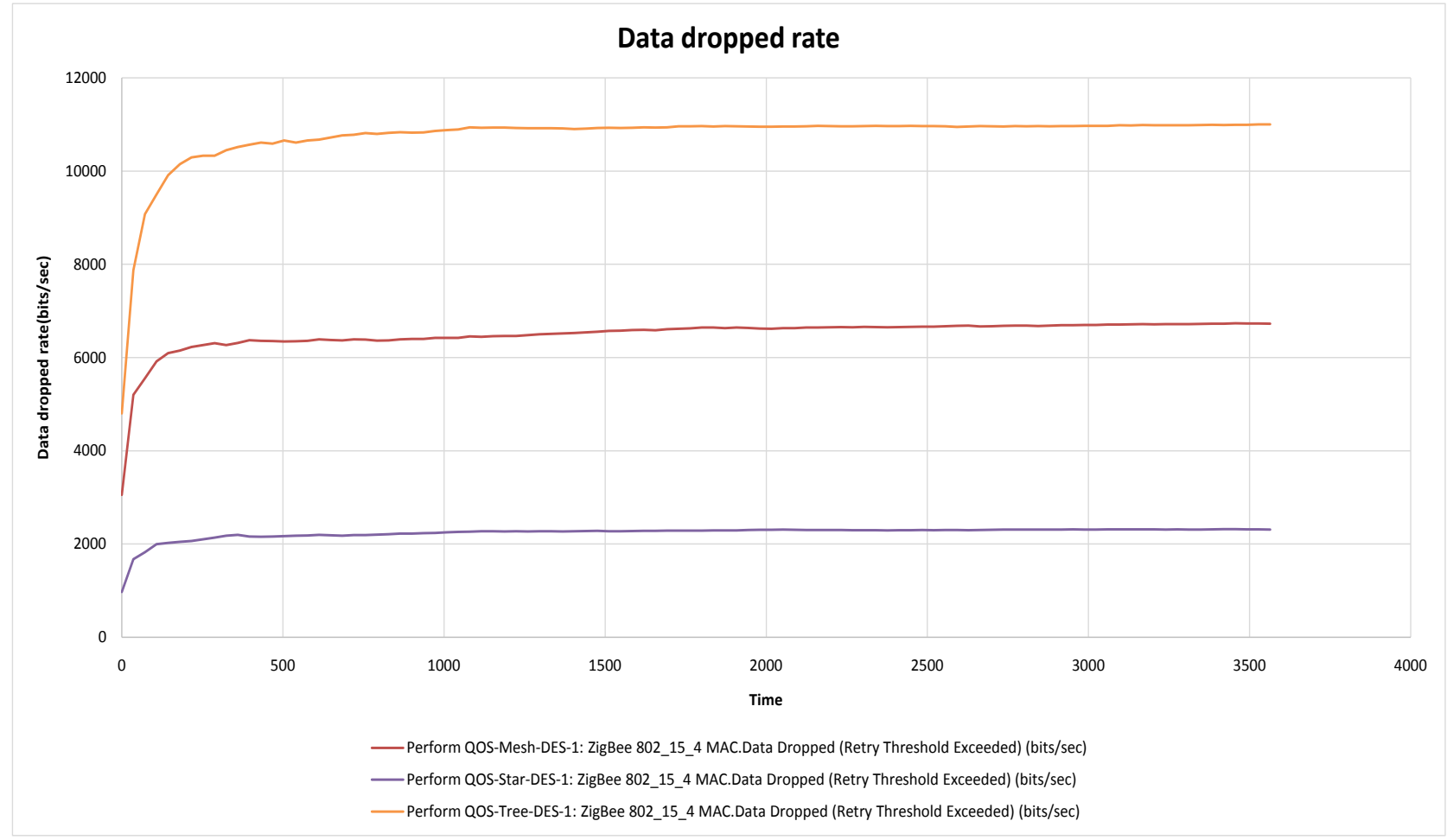

Figure 4. Data dropped rate (bits/second) for scenario 1.

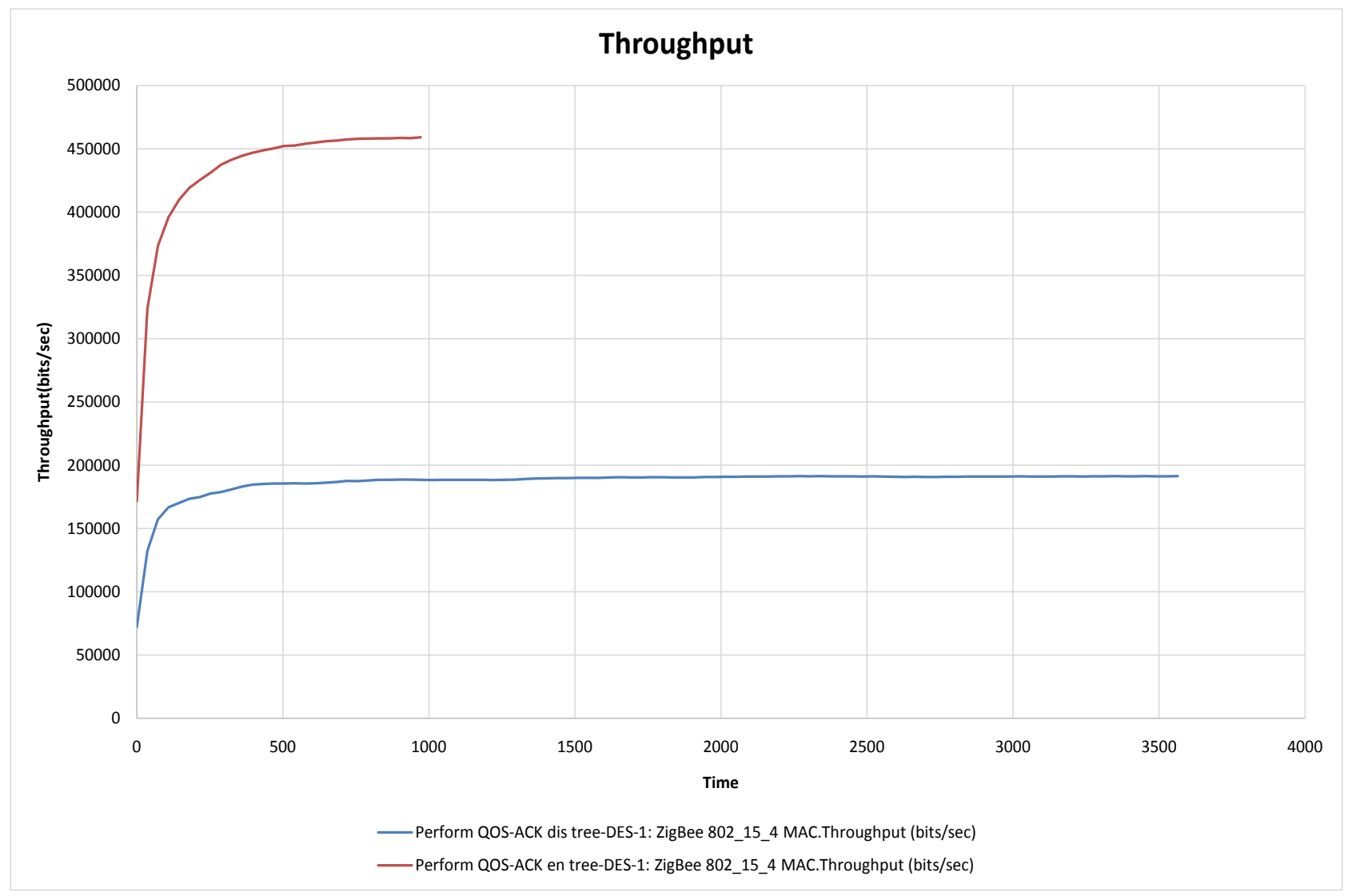

Figure 5. Throughput (bits/second) for scenario 2. 


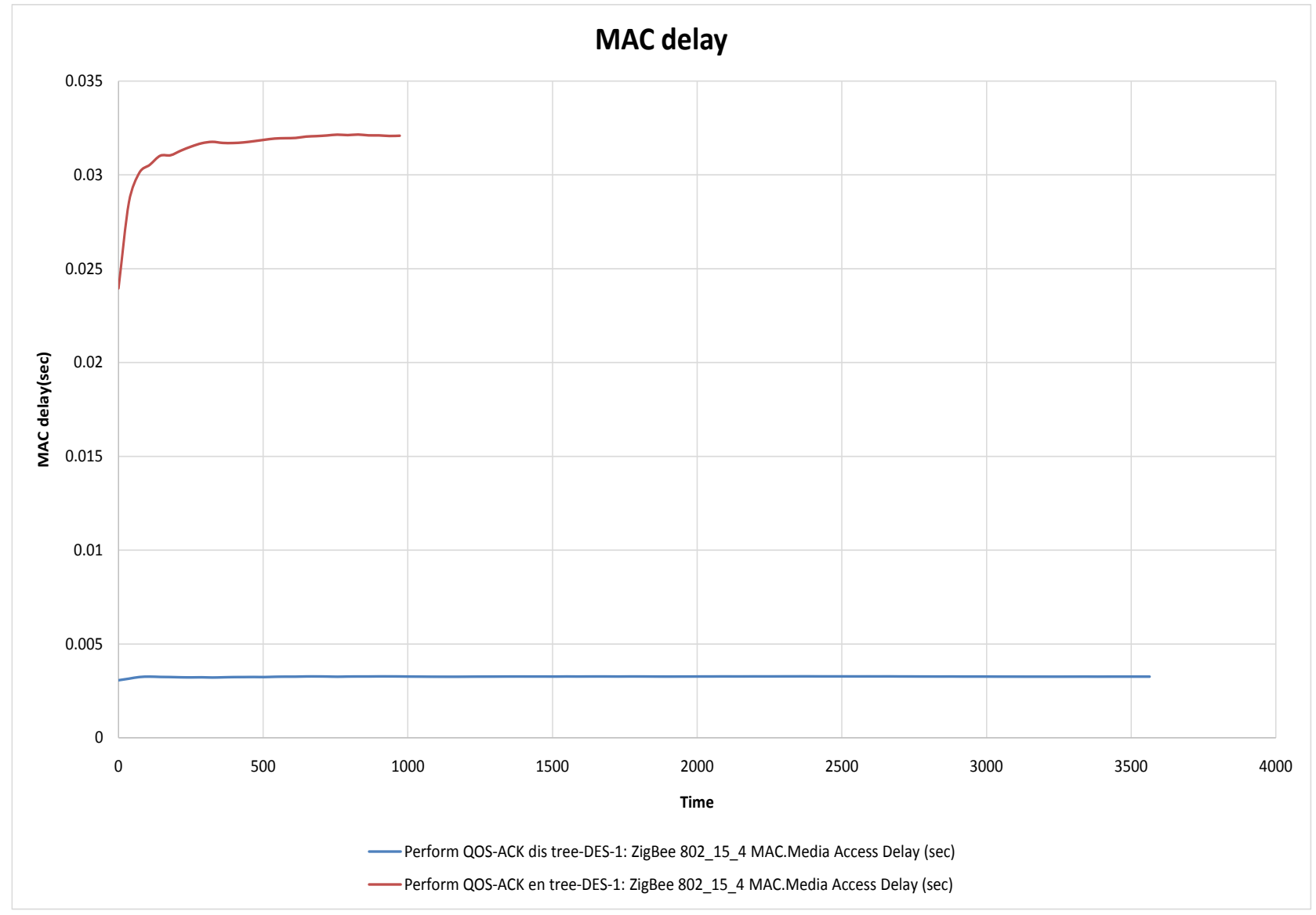

Figure 6. MAC delay (second) for scenario 2.

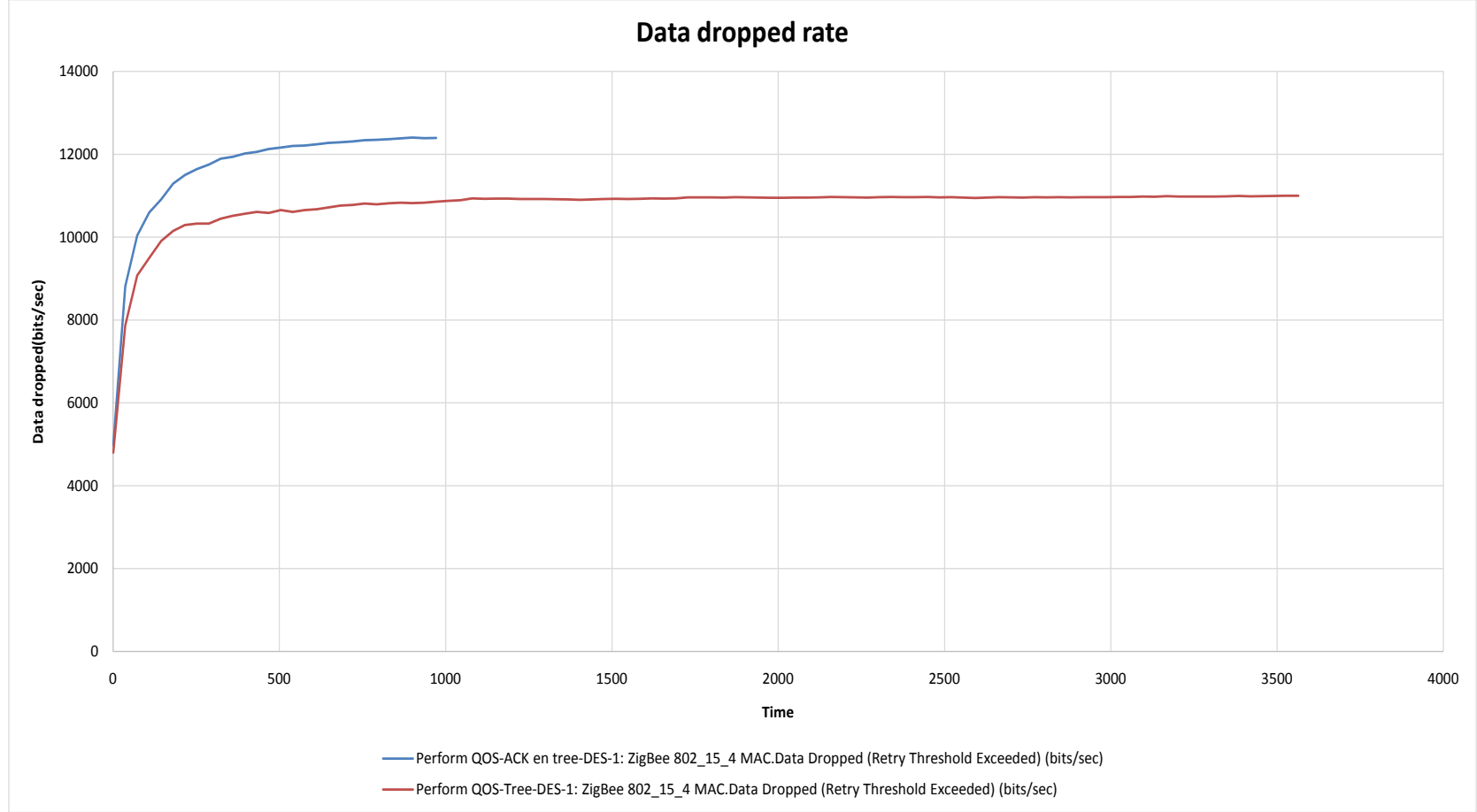

Figure 7. Data dropped rate (bits/second) for scenario 2. 


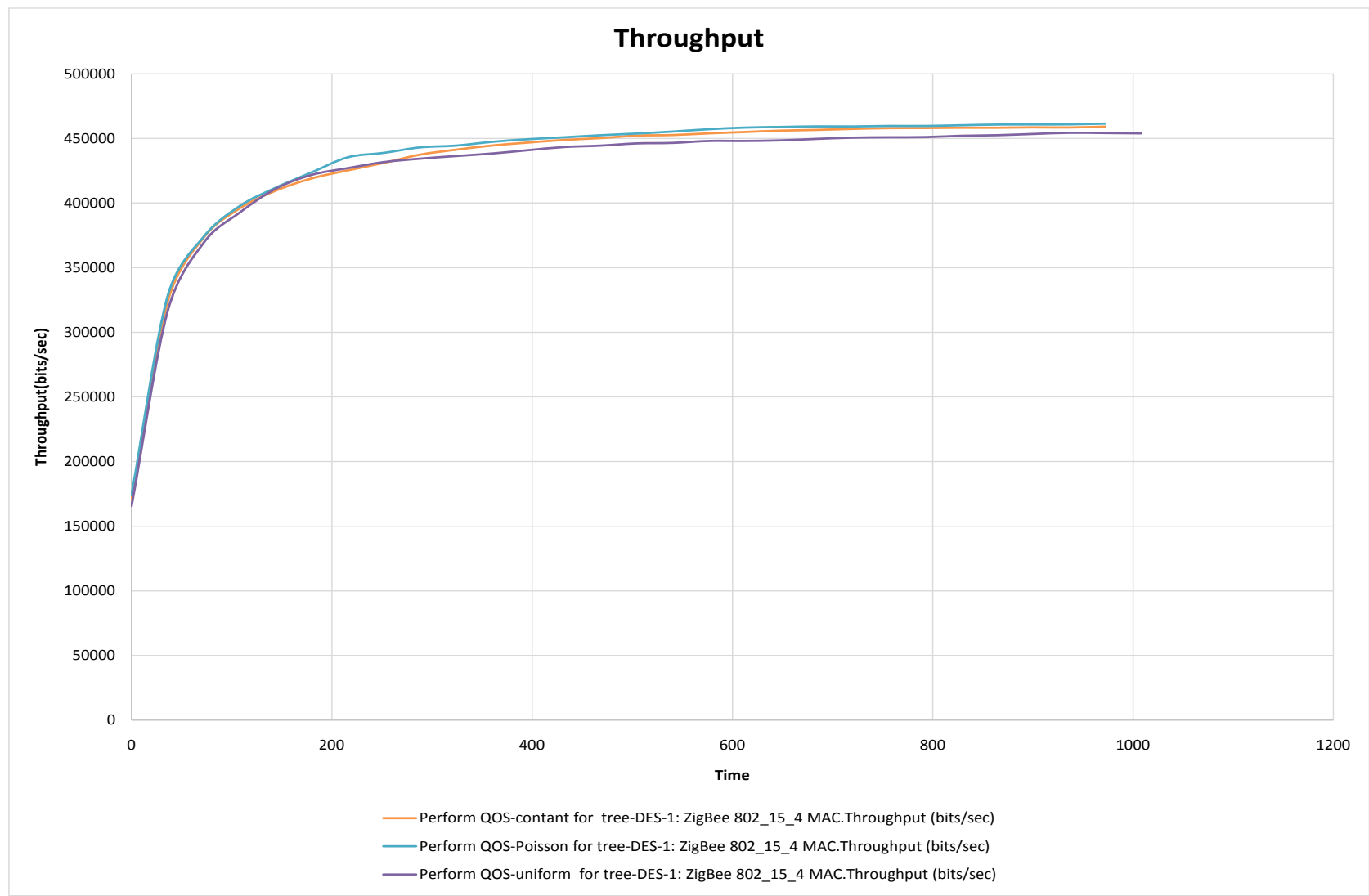

Figure 8. Throughput (bits/second) for scenario 3.

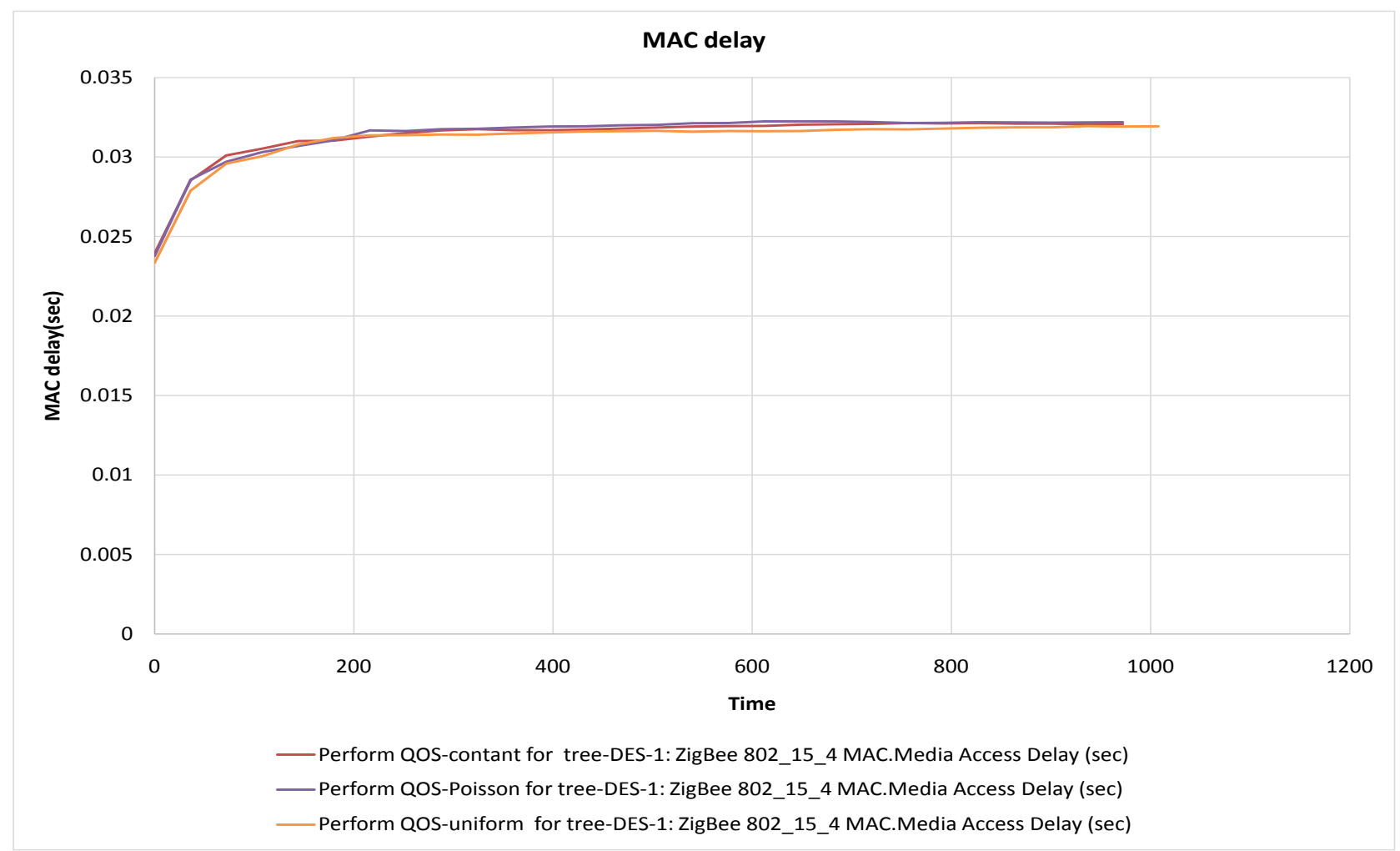

Figure 9. MAC delay (second) for scenario 3. 


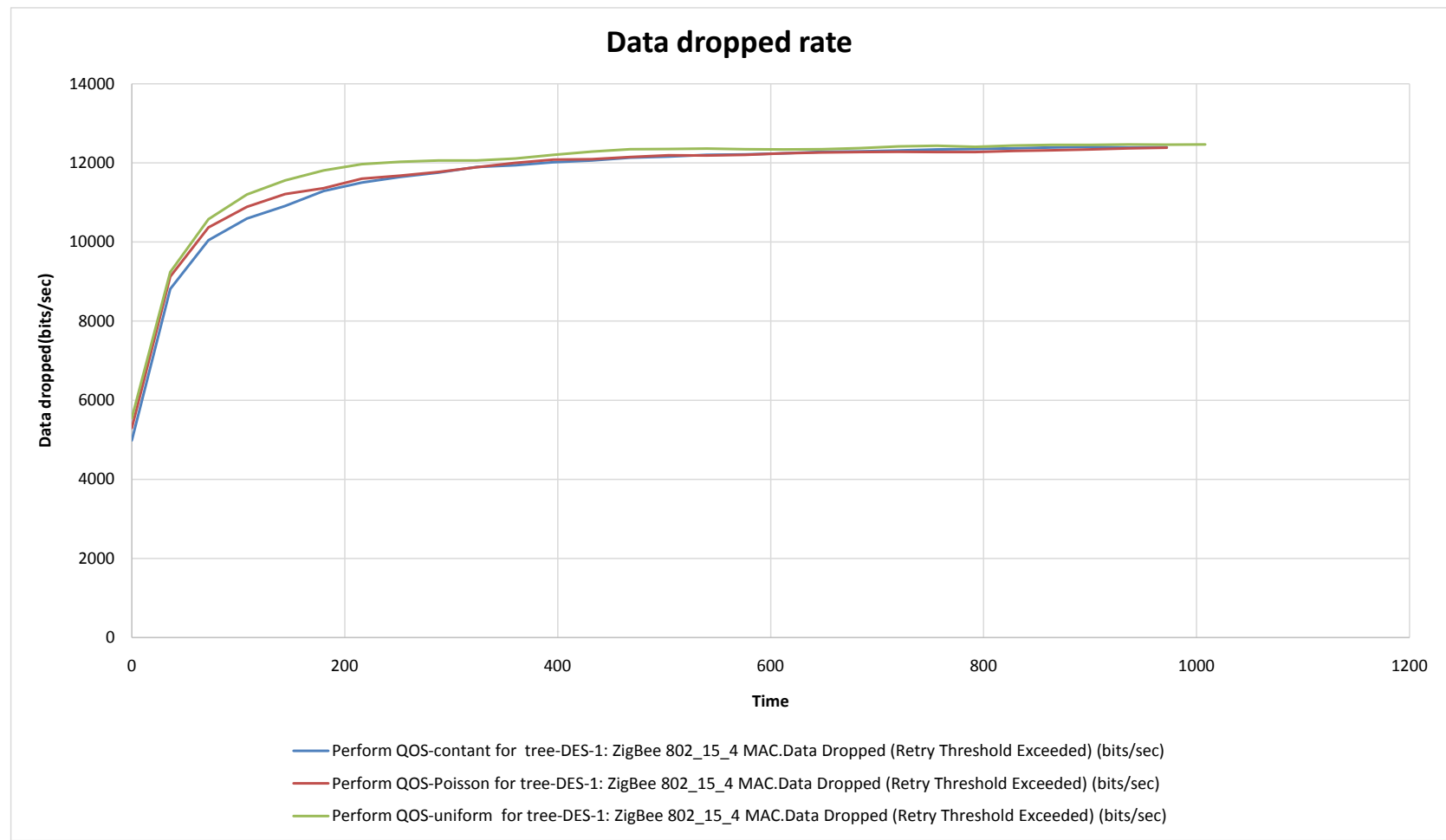

Figure 10. Data dropped rate (bits/second) for scenario 3.

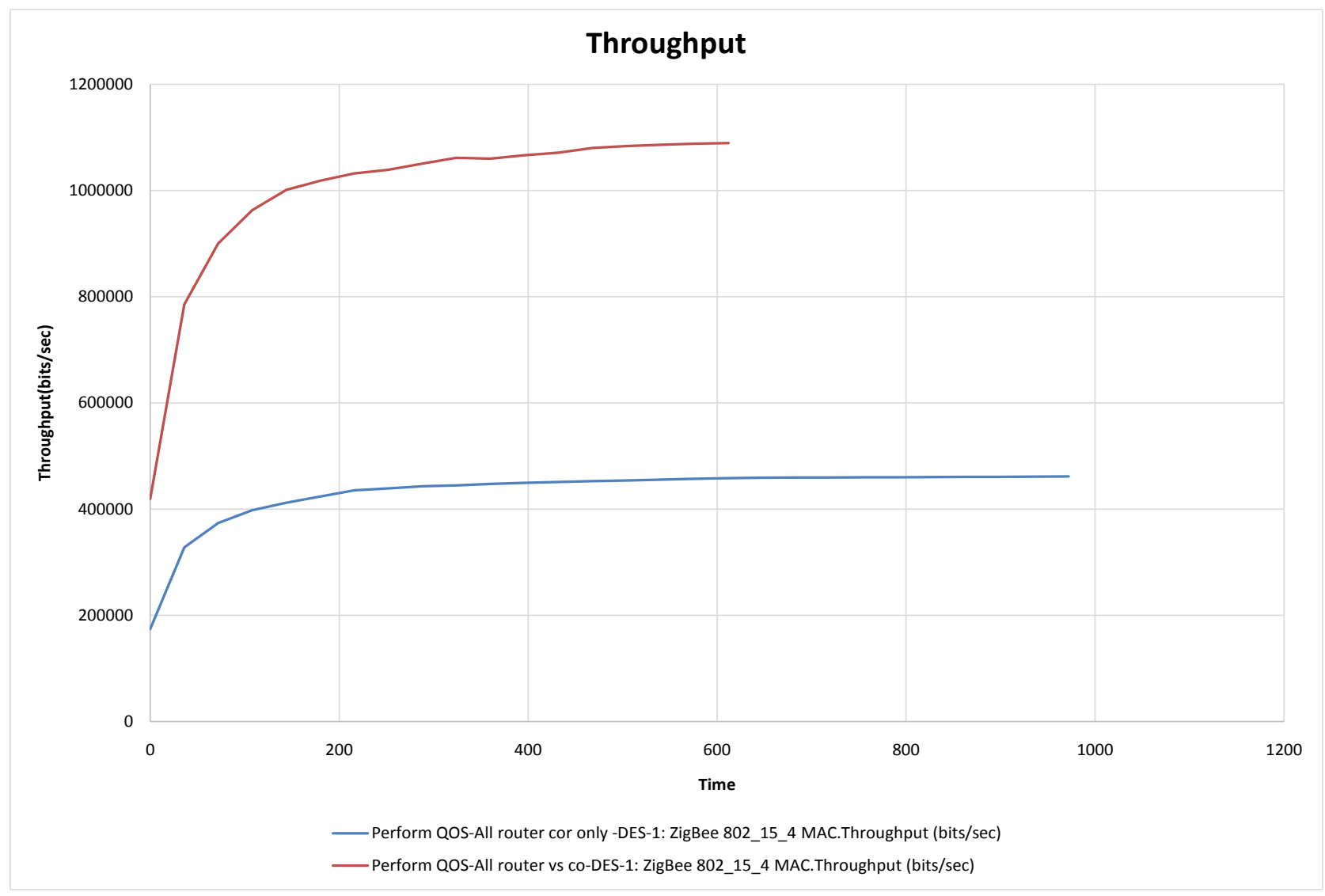

Figure 11. Throughput (bits/second) for scenario 4. 


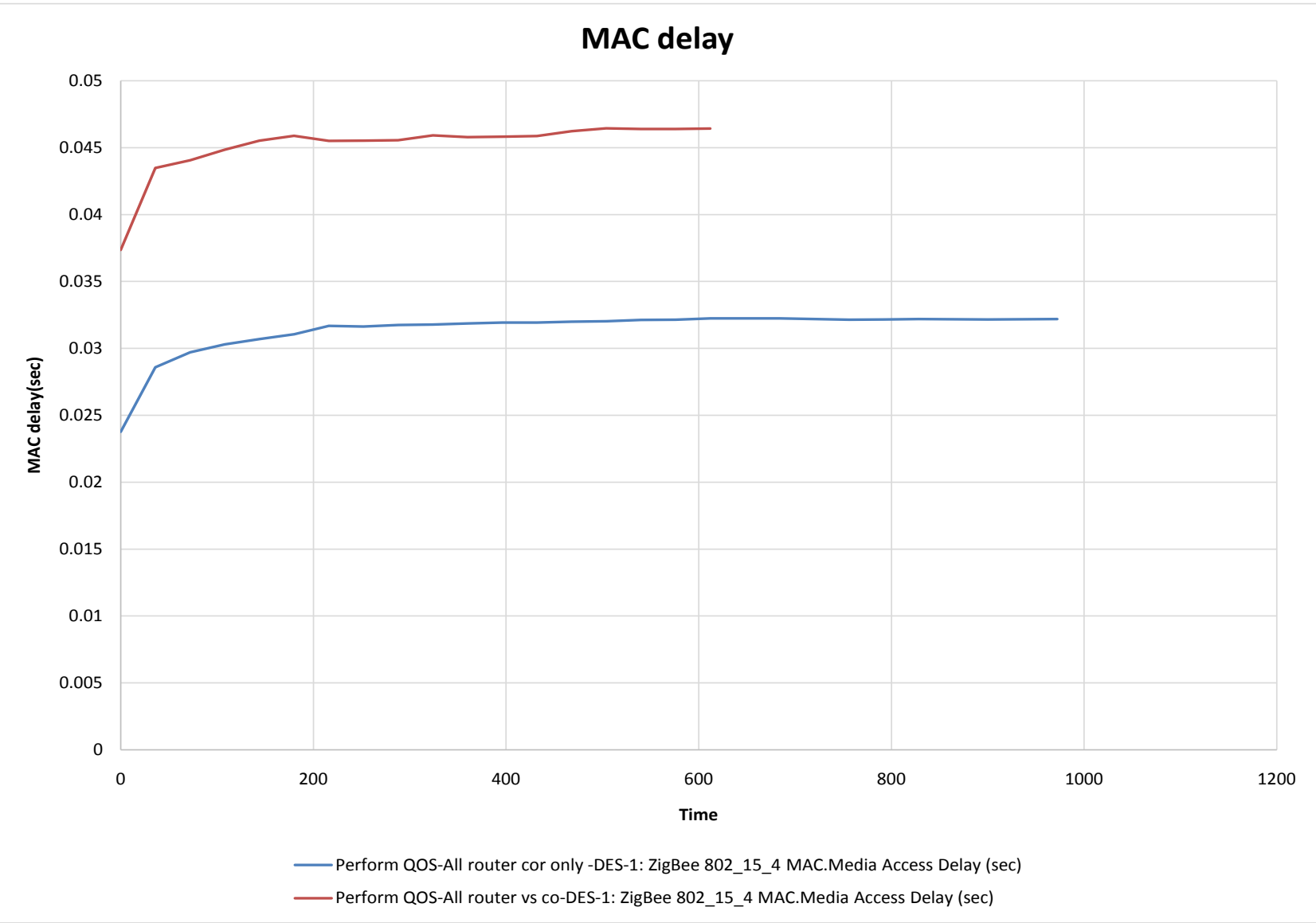

Figure 12. MAC delay (second) for scenario 4.

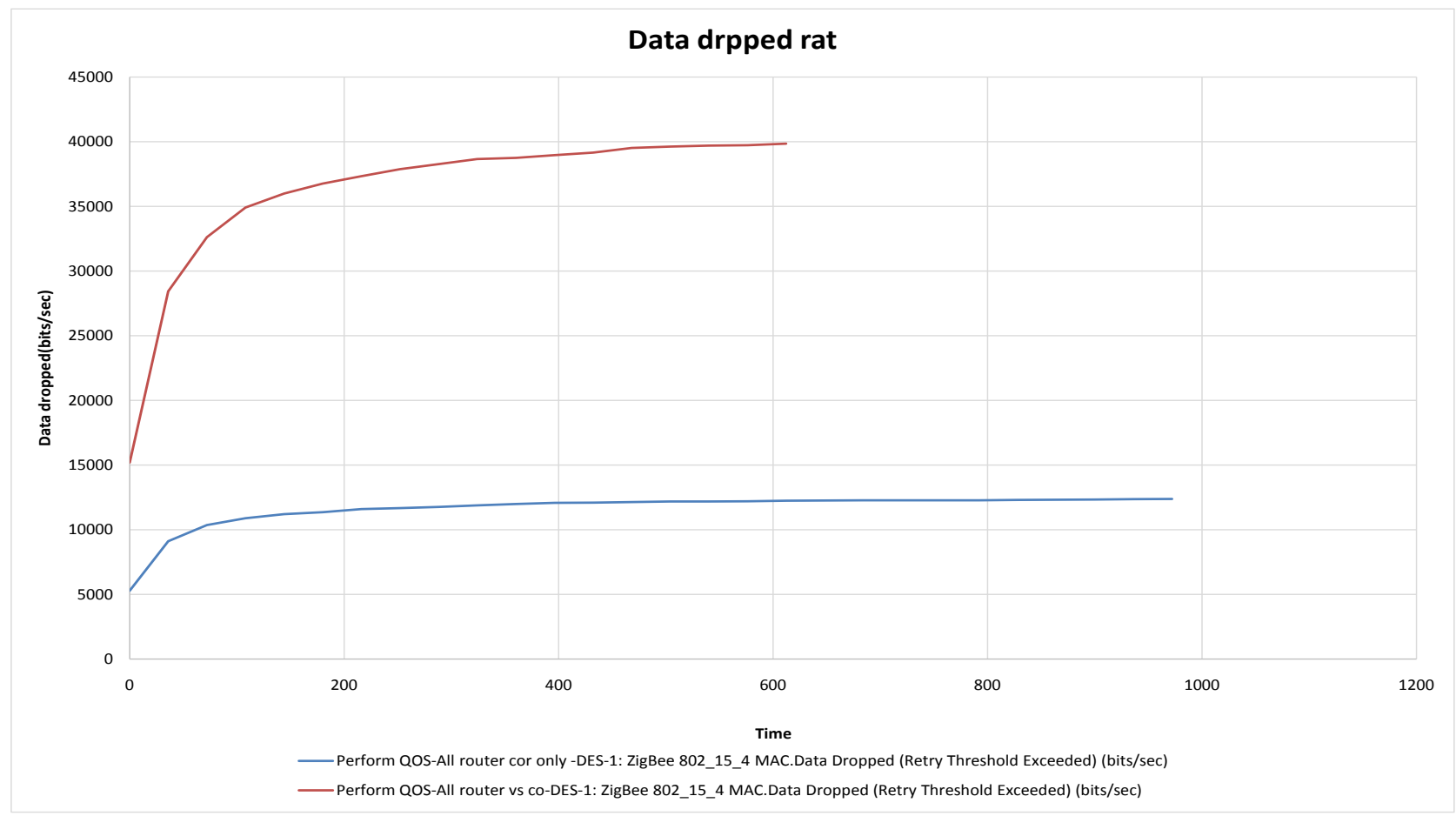

Figure 13. Data dropped rate (bits/second) for scenario 4. 


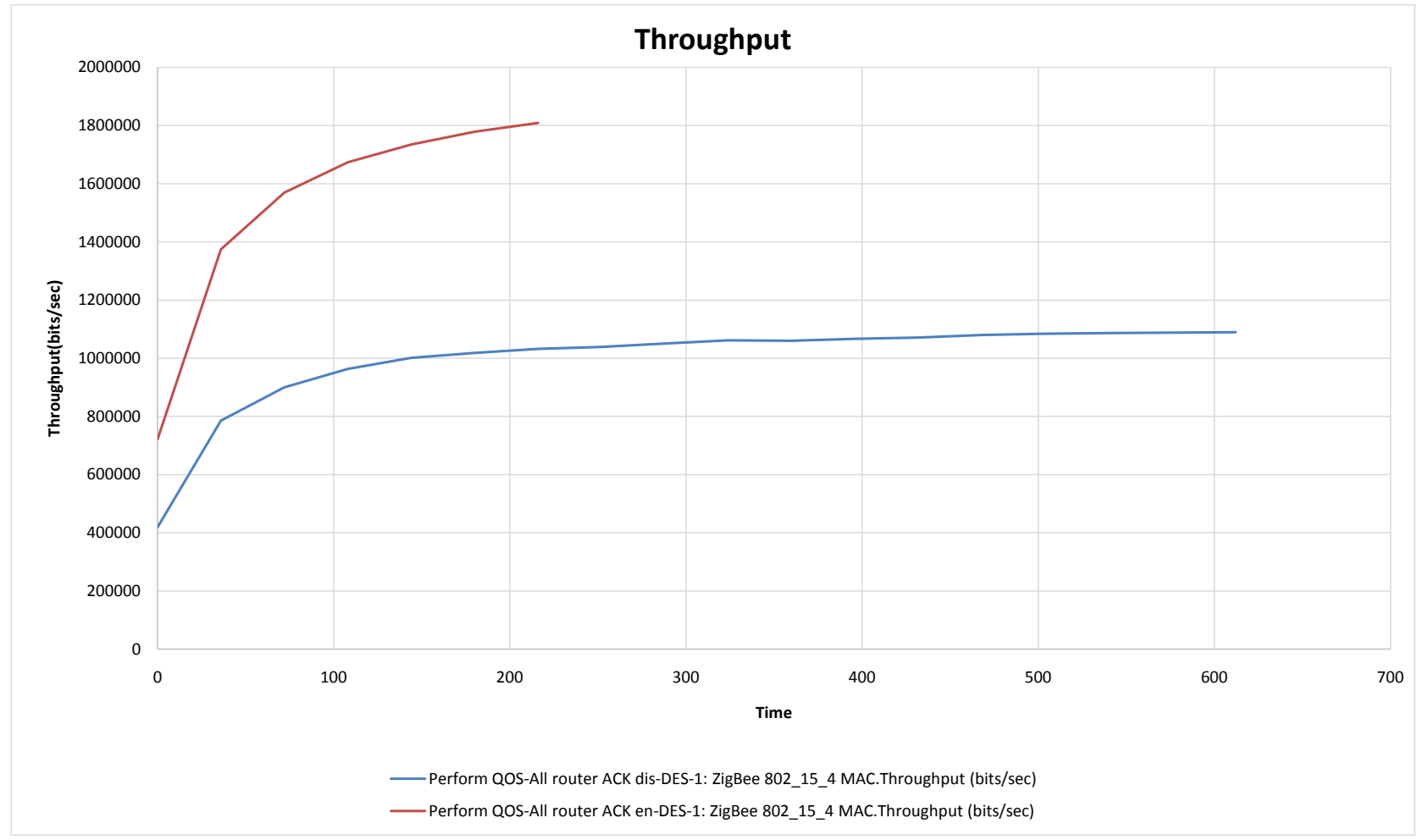

Figure 14. Throughput (bits/second) for scenario 5.

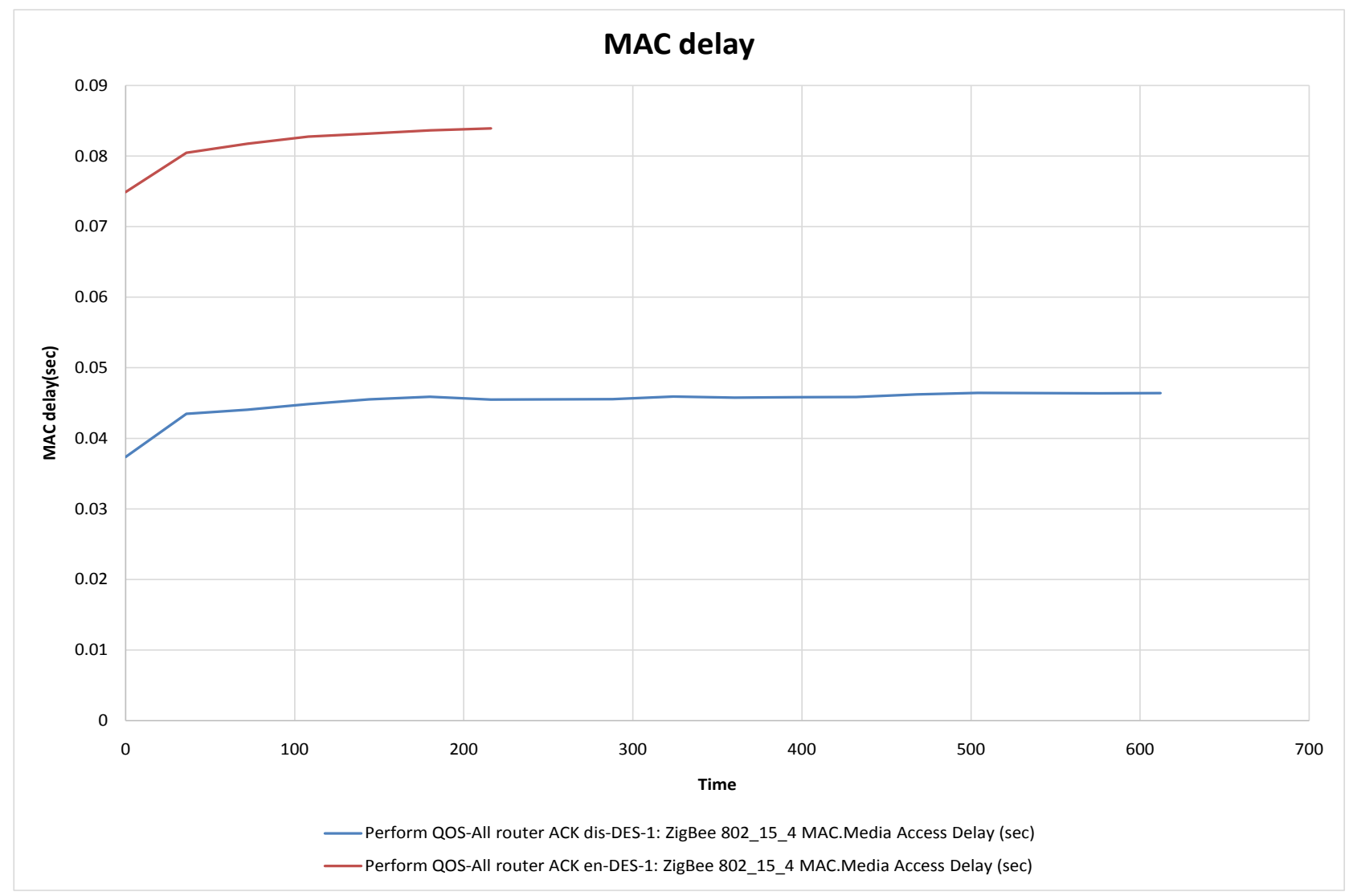

Figure 15. MAC delay (second) for scenario 5. 


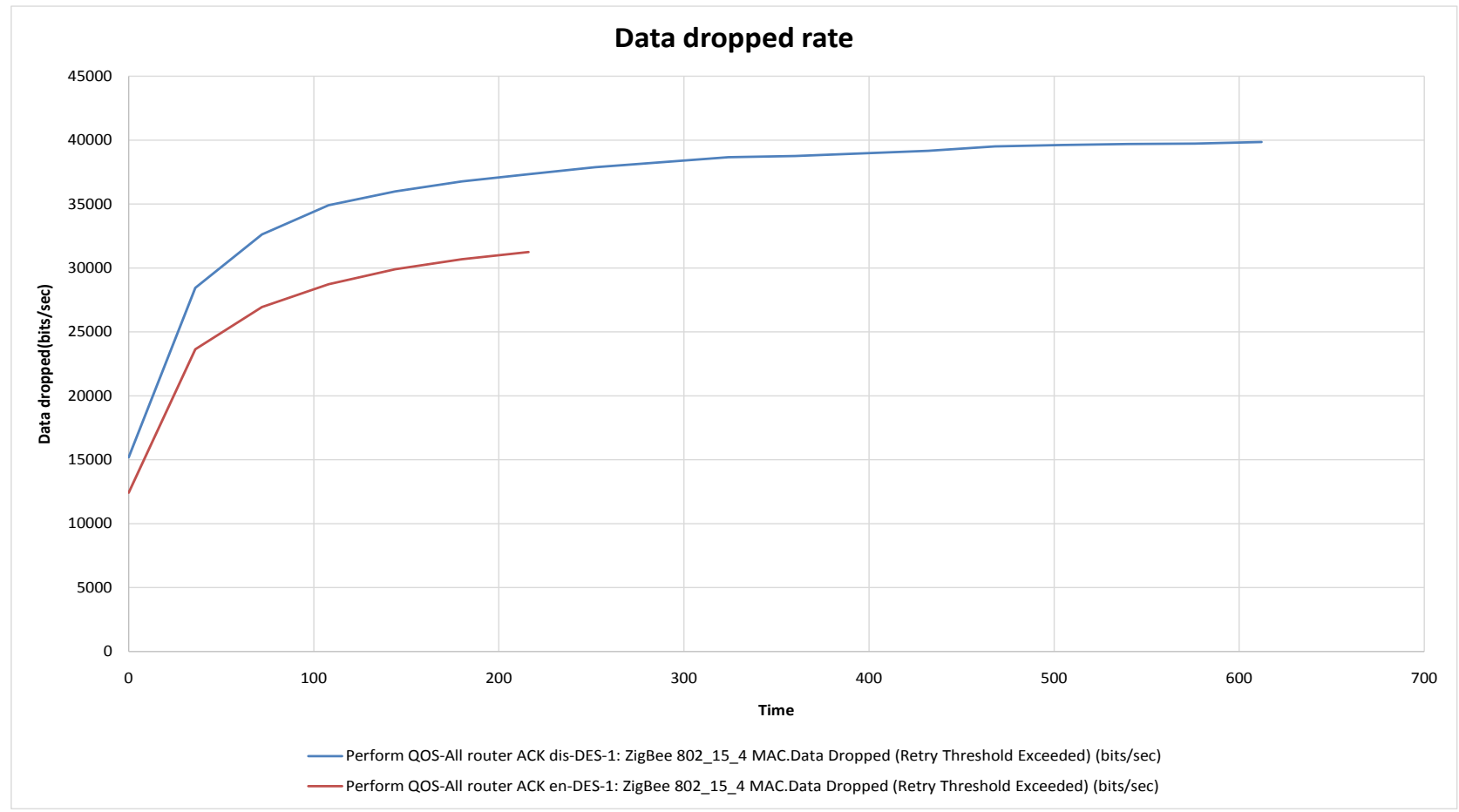

Figure 16. Data dropped rate (bits/second) for scenario 5.

Table 2. Comparative performance chart.

\begin{tabular}{cccc}
\hline Scenario & $\begin{array}{c}\text { Average Throughput } \\
\text { (bits/second) }\end{array}$ & $\begin{array}{c}\text { Average MAC delay } \\
\text { (second) }\end{array}$ & $\begin{array}{c}\text { Average Data dropped rate } \\
\text { (bits/second) }\end{array}$ \\
\hline Scenario 1 & 186,631 & 0.003257 & $10,743.3$ \\
Scenario 2 & $428,941.6$ & 0.031319 & $10,743.3$ \\
Scenario 3 & $432,023.2$ & 0.031393 & $11,620.1$ \\
Scenario 4 (Optimized) & $994,525.6$ & 0.04517 & 3619 \\
Scenario 5 & $1,523,458$ & 0.081525 & $26,228.79$ \\
\hline
\end{tabular}

data dropped rate it shows lower performance. Since we focused mainly on better throughput, this scenario can be optimized.

For the proper validation of optimization we have selected another scenario in which ACK status was enabled in all coordinators and routers. We found that (in Figures 14-16) with the selected parameters of scenario 5 it shows little higher throughput but in terms of average MAC delay and average data dropped rate, the performance decreases.

Finally we can say that in the scenario 4 with tree routing, ACK status enabled coordinator, Poisson packet distribution and routers' destination to all coordinators and routers show the optimized performance. Therefore this configuration will result in better Quality of Service (QoS) (Table 2).

\section{Conclusions}

This research sketches a network designing flow to get high throughput, low MAC delay and low data dropped rate. An extensive simulation was performed 
to get the result. The simulation results illustrate that the optimized scenario shows 5.3 times higher throughput than the first scenario. In addition, data dropped rate reduces to approximately one-third of the first scenario. Though in terms of average MAC delay there need some improvements.

Future work includes aspect of Quality of Experience (QoE) of the designed ZigBee network. In addition, load balancing issues as well as reduction of MAC delay of the network can be studied.

\section{References}

[1] Karl, H. and Willig, A. (2007) Protocols and Architectures for Wireless Sensor Networks. John Wiley \& Sons.

[2] Estrin, D., Girod, L., Pottie, G. and Srivastava, M. (2001) Acoustics, Speech, and Signal Processing. ICASSP01, IEEE International Conference on Instrumenting the World with Wireless Sensor Networks, 4, 2033-2036.

[3] Zheng, J. and Lee, M.J. (2004) Will IEEE 802.15. 4 Make Ubiquitous Networking a Reality?: A Discussion on a Potential Low Power, Low Bit Rate Standard. IEEE Communications Magazine, 42, 140-146. https://doi.org/10.1109/MCOM.2004.1304251

[4] Gislason, D. (2008) ZigBee Wireless Networking. Newness.

[5] Yang, C.T., Chen, S.T., Chang, C.H., Den, W. and Wu, C.C. (2018) Implementation of an Environmental Quality and Harmful Gases Monitoring System in Cloud. Journal of Medical and Biological Engineering, 1-14. https://doi.org/10.1007/s40846-018-0383-0

[6] Biddut, M.J.H., Islam, N., Karim, M.M. and Miah, M.B.A. (2016) An Analysis of QoS in ZigBee Network Based on Deviated Node Priority. Journal of Electrical and Computer Engineering, 3. https://doi.org/10.1155/2016/6254395

[7] Islam, N., Biddut, M.J.H., Arif, M.F.H., Rahman, M.M. and Rahman, M.S. (2016) Mobility Issue on Octagonal Structured ZigBee Network Using Riverbed. International Journal of Communications, Network and System Sciences, 9, 55. https://doi.org/10.4236/ijcns.2016.93005

[8] Lavric, A., Popa, V., Males, C. and Finis, I. (2012) A Performance Study of ZigBee Wireless Sensors Network Topologies for Street Lighting Control Systems. International Conference on Selected Topics in Mobile and Wireless Networking (iCOST), 130-133. https://doi.org/10.1109/iCOST.2012.6271280

[9] Zheng, J. and Lee, M.J. (2006) A Comprehensive Performance Study of IEEE 802.15.4. Sensor Network Operations, 4, 218-237.

[10] Moridi, M.A., Kawamura, Y., Sharifzadeh, M., Chanda, E.K., Wagner, M. and Okawa, H. (2018) Performance Analysis of ZigBee Network Topologies for Underground Space Monitoring and Communication Systems. Tunnelling and Underground Space Technology, 71, 201-209. https://doi.org/10.1016/j.tust.2017.08.018

[11] Islam, N., Biddut, M.J.H., Swapna, A.I. and Asaduzzaman, S. (2015) Improved Quality of Service in ZigBee Network with Statistical Modeling. International Conference on Advances in Electrical Engineering (ICAEE), 174-177.

[12] Sun, T., Liang, N.C., Chen, L.J., Chen, P.C. and Gerla, M. (2007) Evaluating Mobility Support in Zigbee Networks. International Conference on Embedded and Ubiquitous Computing. Springer, Berlin, 87-100.

https://doi.org/10.1007/978-3-540-77092-3_9 
[13] Biddut, M.J.H., Arif, M.F.H. and Islam, N. (2017) Queue Management of RED Enabled ZigBee Network Based on Packet Size Variations and Distribution Techniques. International Conference on Electrical, Computer and Communication Engineering (ECCE), 798-802. 